\title{
Same-sex sexual behavior and selection for indiscriminate mating
}

\author{
Brian A Lerch* and Maria R Servedio
}

Department of Biology. University of North Carolina at Chapel Hill.

250 E Franklin St. Chapel Hill, NC 27514.

*blerch@live.unc.edu 

to pose an evolutionary conundrum ${ }^{1-3}$, as participants in SSB suffer the cost of failing to reproduce after expending the time and energy to find a mate. The potential for SSB to occur as part of an optimal strategy has received almost no attention, although indiscriminate sexual behavior may be the ancestral mode of sexual reproduction ${ }^{4}$. Here, we build a simple model of sexual reproduction and create a theoretical framework for the evolution of indiscriminate sexual behavior. We provide strong support for the hypothesis that SSB is likely maintained by selection for indiscriminate sexual behavior, by showing that indiscriminate mating is the optimal strategy under a wide range of conditions. mating were likely present at the origin of sexual behavior. These findings have implications not only for the evolutionary origins of SSB, but also for the evolution of discriminate sexual behavior across the animal kingdom. activity between two or more members of the same sex) in animals are widespread, with evidence of SSB in mammals ${ }^{5-9}$, birds $^{10-14}$, arthropods ${ }^{15-19}$, mollusks $^{20-22}$, echinoderms ${ }^{23-25}$, and other animals ${ }^{26-30}$. Since SSB is traditionally thought to be deleterious, as same-sex matings require energy expenditure but cannot produce offspring, there has been much interest in understanding its origin and maintenance ${ }^{1-5}$. Despite this, there exists no strong theoretical

20 foundation for understanding SSB (but see ${ }^{31,32}$ ), resulting in a wide range of untested verbal 21 arguments in the literature ${ }^{1-5}$. arguing that rather than trying to understand its presence, a more salient question would be to 
24 understand its absence. They hypothesize that indiscriminate sexual behavior (i.e., mating

25 without determining the sex of one's partner) is the ancestral condition, realizing that

26 discriminate sexual behavior (i.e., directing sexual behavior at members of the opposite sex)

27 must evolve through mechanisms controlling sexual signaling and mate choosiness. Of course,

28 the existence of indiscriminate mating as the ancestral condition does not explain its current

29 prevalence ${ }^{33}$. While in some cases (e.g., broadcast spawning and wind pollination) indiscriminate

30 mating predominates as a result of little potential benefit to (or opportunity for) sexual

31 discrimination, it is oftentimes unclear why indiscriminate mating persists. indiscriminate sexual behavior (or prevent the evolution of sexual discrimination) under a wide range of conditions observed in nature. We create a theoretical framework for understanding the conditions that favor indiscriminate sexual behavior and provide a test of whether SSB is likely to result from selection for indiscriminate sexual behavior. We start with a simple optimization model of sexual reproduction, then support this approach with a population genetic model that explicitly tracks evolutionary dynamics. We find that indiscriminate mating is the optimal strategy for many parameter combinations and produce testable predictions about the conditions that favor SSB resulting from indiscriminate mating.

\section{Optimization Model} basic summary of its features here. Our approach explores one of many ${ }^{34}$ potential hypotheses

44 for SSB (that it results from indiscriminate mating) without considering the evolution of samesex preferences that have evolved in some vertebrates and may result from complex social or genetic interactions (see Table 2 in Bailey and $\mathrm{Zuk}^{1}$ ). As a result, and because our model does 
47 not make assumptions consistent with sexual behavior in humans, this study should not be

48 considered in relation to human sexuality. We assume that a population consists of two sexes

49 (the searching sex and the targeted sex), where a proportion $\sigma$ is of the targeted sex. We make no

50 assumptions about the identity of the sexes and use the terms searching and targeted liberally.

51 For example, if our model were applied to an insect in which males seek females to display to,

52 males would be the searching sex and the females would be the targeted sex.

We assume that reproduction occurs in discrete bouts (corresponding to generations)

54 where each member of the searching sex has only one opportunity to mate per bout (an

55 assumption that biases against indiscriminate mating since SSB cannot be corrected for within

56 one reproductive bout). We assume that an individual of the searching sex finds another

57 individual of either sex with which to attempt to mate with probability $f$. The evolutionarily

58 labile parameter of our model $a$ controls whether the searching sex attempts to mate

59 discriminately. In particular, $a$ is the proportion of bouts in which a member of the searching sex

60 attempts to sexually discriminate. Of course, members of the searching sex can only mate

61 discriminately if they identify some signal (or cue) that an individual is of the opposite sex. We

62 define $s$ as the proportion of bouts in which a member of the targeted sex provides a signal of

63 their sexual identity. Then, as shown in the Supplementary Methods, given that a member of the

64 searching sex finds a mate, it will be of the opposite (targeted) sex with probability $\sigma+(1-\sigma) a s$.

65 Thus, if members of the targeted sex always signal $(s=1)$ and members of the searching sex

66 always attempt to discriminate $(a=1)$, a member of the searching sex is guaranteed to find a

67 member of the targeted sex. Furthermore, without any signal from the targeted sex $(s=0)$ or any

68 attempt to discriminate from the searching sex $(a=0)$, the probability of finding a mate of the 
opposite sex is simply the proportion of the population of that sex $\sigma$. We discuss the interpretation of $a$ and $s$ further in the Supplementary Methods.

72 potential mate (with probability $r$ ), in which case they do not reproduce in the reproductive bout.

73 We assume that matings suffer a fecundity cost $p$ associated with the sexual signal. Individuals

74 from the searching sex die between reproductive bouts with probability $d$ in the absence of

75 sexual discrimination. They also carry an additional survival cost $c$ when they attempt sexual

76 discrimination (a search cost), such that a member of the searching sex will survive to the next

77 reproductive bout with probability $1-(d+a c)$.

\section{Analysis and Results}

The model above results in a wide range of parameter space in which indiscriminate

81 reproductive success of a member of the searching sex, $R_{0}$. Differentiating $R_{0}$ with respect to $a$

82 gives the fitness gradient $\mathrm{d} R_{0} / \mathrm{d} a$ (see Supplementary Methods). At a given amount of sexual

83 discrimination $a$, the sign of the fitness gradient gives the expected direction of evolution. Values

84 of $a$ for which the fitness gradient is 0 are potential evolutionary optima. In analyzing the

85 optimal amount of sexual discrimination, one can determine under what conditions, if any,

86 individuals should attempt to mate indiscriminately. If the optimal strategy is indiscriminate

87 mating, then SSB is expected to be frequent.

Of particular interest is whether indiscriminate mating $(a=0)$ is ever an optimal strategy.

89 We show in the Supplementary Methods that the fitness gradient at $a=0$ will be negative (and

90 thus sexual discrimination should never evolve) whenever 


$$
c>(1-\sigma)(1-d) d s / \sigma
$$

92 Given a 1:1 sex ratio $(\sigma=0.5)$, equation (1) simplifies to $c>(1-d) d s$. Equation (1) shows that

93 even under restrictive conditions in which the targeted sex always provides a sexual signal $(s=$

94 1), the optimal strategy may be to never attempt sexual discrimination. Without sexual signaling

$95(s=0)$, if there is any cost to attempting to sexually discriminate, sexual discrimination is not

96 expected to evolve. Although this is obvious given the formulation of the model, it formalizes

97 the important point that the origin of sex and the origin of providing signals of one's sex are not

98 the same. Logically, such cues likely evolved after the origin of sexual reproduction ${ }^{4}$, so our model suggests selection for sexual discrimination was unlikely to follow immediately upon the

100 origin of sex, strengthening the hypothesis that indiscriminate sexual behavior is likely

101 ancestral ${ }^{4}$.

Similarly, the conditions for maximum attempted discrimination $a=1$ to be the best small but non-trivial portion of the parameter space). 
Interestingly, an intermediate death rate $d$ favors the evolution of sexual discrimination

113 (Fig. 1). When death is rare ( $\operatorname{small} d$ ), members of the searching sex are expected to have many

114 reproductive opportunities in their lifetime. Under these conditions, the best strategy is to live as

115 long as possible by not attempting to sexually discriminate. The cost of SSB in this case is low

116 because one failed mating due to SSB will likely be made up for by chance later in life. On the

117 other hand, when $d$ is high, members of the searching sex are unlikely to ever mate. In this case,

118 they cannot afford to pay any additional cost and their optimal strategy is to mate

119 indiscriminately and rely on luck. Of course, indiscriminate mating will result in SSB being

120 common (Fig. 1, bottom row).

Although they do not affect the optimal level of discrimination, increasing the cost of sexual signals $p$ and the probability of mate rejection $r$ and decreasing the probability of finding

123 any individual $f$ cause the selection gradient to approach 0 (i.e., weaker selection; Movie S1 and

124 Table 2 show the effect of each parameter). If indiscriminate mating is ancestral, these conditions

125 are more conducive to the transient maintenance of indiscriminate sexual behavior by reducing

126 the efficacy of selection and making the stochastic loss of discriminate mating more likely. Thus,

127 discriminate mating is less likely to evolve in sparse populations (low $f$ ) or when the targeted sex

128 is choosy or the searching sex competitive (high $r$ ).

We test the generality of our results by modifying our assumptions to allow same-sex matings to carry an additional cost (Supplementary Appendix S1), to include mortality from different sources acting multiplicatively (Supplementary Appendix S2), to assume the cost to

132 sexual discrimination is due to fecundity as opposed to survival (Supplementary Appendix S3), 133 and to assume a semelparous life history (Supplementary Appendix S4). We show the conditions 134 for no or complete sexual discrimination to evolve given these assumptions in Table 1 . We 
135 consider the existence of additional costs to SSB as an extension since while such costs have

136 been found ${ }^{35}$ (and are often suggested ${ }^{3}$ ), other studies fail to support that such costs exist ${ }^{36,37}$.

137 Our primary results are robust to all of these changes, with each version of the model predicting

138 an appreciable region of parameter space for which indiscriminate mating is the optimal strategy.

139 Of course, assuming that SSB carries explicit costs (in addition to the opportunity costs implicit

140 in the above analysis) results in more restrictive conditions for sexual discrimination to evolve,

141 although small costs to SSB have only small impacts on the model outcomes. The only

142 qualitative differences between the model versions occur at high death rates $d$ when mortality is

143 multiplicative, high signaling costs $p$ when discrimination cost is to fecundity, or low death rates

$144 d$ when the searching sex is semelparous. Qualitative outcomes of the models are compared in

145 Table 3.

146 Dynamical Model

Although our optimization model is analytically tractable and clarifies costs and benefits,

148 lifetime reproductive success is not necessarily maximized by selection ${ }^{38}$. As such, we also build 149 a single-locus population genetic model with haploid genetics and overlapping generations that 150 makes similar assumptions to the approach above. Importantly, the population genetic model 151 extends the optimization approach by incorporating frequency dependence and allowing the sex 152 ratio to change naturally from feedbacks with mortality due to discrimination costs. Since $p, r$, 153 and $f$ play no role in this framework, they are ignored. We still assume a background mortality of $154 d$ afflicts both sexes and a survival cost of attempted discrimination of $a c$ is suffered only by the 155 searching sex. The probability of finding a mate of the opposite sex is still $\sigma+(1-\sigma) a s$, but now 156 the sex ratio $\sigma$ emerges from the model. We use successive invasions to determine the 
157 evolutionarily stable values of attempted sexual discrimination $a$. Although not analytically

158 tractable, this model makes no assumptions a priori about the quantity that selection maximizes.

The results from this population genetic model align strikingly well with the optimization approach, with the range of conditions under which indiscriminate mating is uninvadible being

161 practically identical between approaches (Fig. S1). A stable polymorphism identified by mutual

162 invasibility only occurs in about $2 \%$ of 10,000 randomly generated parameter combinations and

163 is especially common at low or high death rates $d$ and strong sexual signals $s$. This model shows

164 that not attempting to discern the sex of potential mates can be a convergent stable evolutionary

165 optimum.

\section{Implications}

SSB is often considered a result of mistaken identity ${ }^{39-41}$, as is suggested to account for

168 about $80 \%$ of reported cases in arthropods ${ }^{3}$. Our model provides an evolutionary perspective on

169 this mistaken identity hypothesis, suggesting that poor sex identification could actually occur as

170 an optimal strategy. This evokes hypotheses that SSB may result from a mating strategy of

171 attempting copulation with any encountered conspecific due to low probability of encounter ${ }^{21}$ or

172 low costs to $\mathrm{SSB}^{22,30}$. The costs of missing an opportunity to mate and of attempting SSB have

173 been discussed ${ }^{42-44}$ in the context of the acceptance cost threshold hypothesis ${ }^{45}$ —a general theory

174 suggesting that erroneous associations (e.g. between mates or cooperative partners) become more

175 likely with poor discrimination ability and low costs to mistaken associations. We provide a

176 formal application of this hypothesis to SSB and show which conditions favor indiscriminate

177 mating. 
It was argued by Parker" that six evolutionary transitions (the "sexual cascade") drove

179 unicellular asexual organisms to become behaviorally-complex, sexual organisms. One such

transition is the evolution of the movement of males toward females before sperm release during

181 external fertilization (an example of sexual discrimination referred to as "female targeting" 46 ).

182 To our knowledge, Parker's model ${ }^{46}$ is the only study addressing whether sexual discrimination

183 evolves. Direct comparison between Parker's model and our model is difficult, but we seem to

184 find more restrictive conditions for the evolution of sexual discrimination, which can be

185 attributed to 1) imperfect signaling (our $s$ ) of the targeted sex (as is likely at the origin of sexual

186 reproduction ${ }^{4}$ ) and 2) a search $\operatorname{cost}^{47,48}$ (our $c$ ) for attempting to sexually discriminate (instead of

187 a tradeoff with gonad expenditure ${ }^{46}$ ). These models are complementary; our model applies to

188 cases not considered by Parker $^{46}$ such as SSB in species with internal fertilization (e.g. insects ${ }^{3}$ )

189 or species with limits on their ability to find and identify mates (e.g. those with search costs, poor

190 signals of sexual identity, and deep-sea species ${ }^{, 21,23,24}$ ).

It is interesting to consider how the predictions of the model relate to the conditions

192 expected at the origin of sexual behavior. Echinoderms are likely good proxies for such

193 animals ${ }^{4,46}$, supported by their position as an outgroup to chordates (where most complexity in

194 sexual behaviors arise). Consistent with the model's predictions for species that mate

195 indiscriminately, long-lived adults are common in echinoderms ${ }^{49,50}$. Additionally, it is reasonable

196 to expect that cues to determine sex in echinoderms are relatively limited both because visual

197 cues cannot be relied upon and there exists little evidence in this taxon for chemical cues for sex-

198 specific recognition from a distance ${ }^{51,52}$. Indeed, multiple studies suggest some echinoderm

199 species form mating pairs without consideration for $\operatorname{sex}^{23,24,53}$. This suggests that if 
200 indiscriminate sexual behavior is the ancestral condition ${ }^{4}$, sexual discrimination was unlikely to 201 have evolved readily.

This model relates to previous work on mate choice in which there can be a direct cost of mating with one category of individuals versus another ${ }^{54}$, in finding that costs can prevent mating preferences from evolving. In fact, all cases where there are direct viability or fecundity benefits to choosing one type of mate are also somewhat analogous. However, the costs of indiscriminate mating in the current model are much higher than in many other cases with direct

207 benefits, as SSB results in a mating which cannot produce any offspring at all. The mechanisms 208 operating here are most similar to the evolution of preferences for conspecifics, where mating 209 with a heterospecific produces no viable hybrids. In both cases, costs of discrimination will trade 210 off against the peril of producing no offspring. In the current context, the unexpected 211 consequence is that SSB often results.

213 can be an optimal strategy, we extend recent work ${ }^{4}$ suggesting the evolutionary origins of

214 discriminate sexual behavior as a new and fruitful area of research. Our model provides an

215 important proof-of-concept regarding whether indiscriminate mating can be an optimal

216 evolutionary strategy and what conditions facilitate its evolution. One important result from this

217 modeling exercise is that sexual discrimination can be favored by either low, intermediate, or

218 high mortality rates depending on other features of the system (Table 3). As such, mortality rates

219 alone are unlikely to predict whether indiscriminate mating is an optimal strategy. Costs to

220 discrimination $c$ and strengths of sexual signals $s$ are more likely candidates for the drivers of

221 indiscriminate mating, but their values in natural populations are unknown. Attempts to measure

222 these (or related) parameters are important gaps to fill in determining whether SSB results from 
selection for indiscriminate mating in nature. Specifically, our model leads to the predictions that species that mate indiscriminately have high costs to discrimination, search costs to survival rather than fecundity (since this results in more stringent conditions for sexual discrimination, Supplementary Appendix S3), and subtle differences between the sexes. In this way, our model suggests which features of organisms are likely to predispose them to indiscriminate mating, providing a guide future work to determine how widespread selection favoring indiscriminate mating is in nature

\section{References}

1. Bailey, N. W. \& Zuk, M. Same-sex sexual behavior and evolution. TREE 24, 439-446 (2009).

2. Caballero-Mendieta, N. \& Cordero, C. Enigmatic liaisons in Lepidoptera: a review of same-sex courtship and copulation in butterflies and moths. J. Insect Sci. 12, 138 (2012).

3. Scharf, I. \& Martin, O. Y. Same-sex sexual behavior in insects and arachnids: prevalence, causes, and consequences. Behav. Ecol. Sociobiol. 67, 1719-1730 (2013).

4. Monk, J. D., Giglio, E., Kamath, A., Lambert, M. R., \& McDonough, C. E. An alternative hypothesis for the evolution of same-sex sexual behavior in animals. Nat. Ecol. Evol. 3, 1622-1631 (2019).

5. Vasey, P. L. Homosexual behavior in primates: A review of evidence and theory. Int. J. Primatol. 16, 173-204 (1995).

6. Vasey, P. L. Same-sex sexual partner preference in hormonally and neurologically unmanipulated animals. Annual Review of Sex Research 13, 141-179 (2002).

7. Vasey, P. L. \& Jiskoot, H. The Biogreography and Evolution of Female Homosexual Behavior in Japanese Macaques. Archives of Sexual Behavior 39, 1439-1441 (2010).

8. Furuichi, T., Connor, R. \& Hashimoto, C. Non-conceptive sexual interactions in monkeys, apes, and dolphins. Primates and Cetaceans, 385-408 (Springer Tokyo, 2013).

9. Sugita, N. Homosexual fellatio: Erect penis licking between male Bonin flying foxes Pteropus pselaphon. PLoS One 11, e0166024 (2016).

10. Lombardo, M. P., Bosman, R. M., Faro, C. A., Houtteman, S. G. \& Kluisza, T. S. Homosexual copulations by male tree swallows. Wilson Ornithological Society 106, 555557 (1994).

11. Zuk, M. Family values in black and white. Nature 439, 917 (2006).

12. MacFarlane, G. R., Blomberg, S. P., Kaplan, G. \& Rogers, L. J. Same-sex sexual behavior in birds: expression is related to social mating system and state of development at hatching. Behav. Ecol. 18, 21-33 (2007). 
13. Young, L. C., Zaun, B. J. \& VanderWerf, E. A. Successful same-sex pairing in Laysan albatross. Biol. Letters 4, 323-325 (2008).

14. MacFarlane, G. R., Blomberg, S. P. \& Vasey, P. L. Homosexual behaviour in birds: frequency of expression is related to parental care disparity between the sexes. Anim. Behav. 80, 375-390 (2010).

15. Harari, A. R., Brockmann, H. J. \& Landolt, P. J. Intrasexual mounting in the beetle Diaprepes abbreviates (L.). PRSB 267, 2071-2079 (2000).

16. Van Gossum, H., De Bruyn, L. \& Stoks, R. Reversible switches between male-male and male-female mating behaviour by male damselflies. Biol. Letters 1, 268-270 (2005).

17. Bailey, N. W., Hoskins, J. L., Green, J. \& Ritchie, M. G. Measuring same-sex sexual behaviour: the influence of the male social environment. Anim. Behav. 86, 91-100 (2013).

18. Martin, F. M., Kruse, K. C. \& Switzer, P. V. Social experience affects same-sex pairing behavior in male red flour beetles (Tribolium castaneum Herbst). J. Insect Behav. 28, 268-279 (2015).

19. Hoskins, J. L., Ritchie, M. G. \& Bailey, N. W. A test of genetic models for the evolutionary maintenance of same-sex sexual behaviour. PRSB 282, 20150429 (2015).

20. Ambrogio, O. V.\& Pechenik, J. A. When is a male not a male? Sex recognition and choice in two sex-changing species. Behav. Ecol. Sociobiol. 62, 1779-1786 (2008).

21. Hoving, H. J. T., Bush, S. L. \& Robison, B. H. A shot in the dark: same-sex sexual behaviour in a deep-sea squid. Biol. Letters 8, 287-290 (2011).

22. Hoving, H. J. T., Fernandez-Alvarez, F. A., Portner, R. J. \& Gilly, W. F. Sex-sex sexual behaviour in an oceanic ommastrephid squid, Dosidicus gigas (Humboldt squid). Mar. Biol. 166, 33 (2019).

23. Young, C. M., Tyler, P. A., Cameron, F. L. \& Rumrill, S. G. Seasonal breeding aggregations in low-density population of the bathyal echinoid Stylocidaris lineata. Mar. Biol. 113, 603-612 (1992).

24. McCarthy, D. A. \& Young, C. M.Gametogenesis and reproductive behavior in the echinoid Lytechinus variegatus. Mar. Ecol. Prog. Ser. 233, 157-168 (2002).

25. Keesing, J. K., Graham, F., Irvine, T. R. \& Crossing, R. Synchronous aggregated pseudocopulation of the sea star Archaster angulatus Muller and Troschel, 1842 (Echinodermata: Asteroidea) and its reproductive cycle in south-western Australia. Mar. Biol. 158, 1163-1173 (2011).

26. Werner, Y. L. Apparent homosexual behavior in an all-female population of a lizard, Lepidodactylus lugubris and its probable interpretation. Ethol. 54, 144-150 (1980).

27. Kazmi, Q. B. \& Tirmizi, N. M. An unusual behavior in box crabs (Decapoda, Brachyura, Calappidae). Crustaceana 53, 313-314 (1987).

28. Nakashima, Y., Sakai, Y., Karino, K. \& Kuwamura, T. Female-female spawning and sex change in a haremic coral-reef fish, Labroides dimidiatus. Zool. Sci. 17, 967-970 (2000).

29. Shine, R. et al. Movements, mating, and dispersal of red-sided gartersnakes (Thamnophis sirtalis parietalis) from a communal den in Manitoba. Copeia 1, 82-91 (2001).

30. Marco, A. \& Lizana, M. The absence of species and sex recognition during mate search by male common toads, Bufo bufo. Ethol. Ecol. Evol. 14, 1-8 (2002). 
31. Gavrilets, S. \& Rice, W. R. Genetic models of homosexuality: generating testable predictions. PRSB 273, 3031-3038 (2006).

32. Rice, W. R., Friberg, U. \& Gavrilets, S. Homosexuality as a consequence of epigenetically canalized sexual development. Q. Rev. Biol. 87, 343-368 (2012).

33. Dickins, T. E. \& Rahman, Q. Ancenstral primacy of same-sex sexual behavior does not explain its stable prevalence in modern populations. Nat. Ecol. Evol. 4, 782-783 (2020).

34. Clive, J., Flintham, E. \& Savolainen, V. Understanding same-sex sexual behaviour requires thorough testing rather than reinvention of theory. Nat. Ecol. Evol. 4, 784-785 (2020)

35. Spratt, E. C. Male homosexual behaviour and other factors influencing adult longevity in Tribolium castaneum (Herbst) and T. confusum Duval. J. Stored Prod. Res. 16, 109-114 (1980).

36. Maklakov, A. A. \& Bonduriansky, R. Sex differences in survival costs of homosexual and heterosexual interactions: evidence from a fly and a beetle. Anim. Behav. 77, 13751397 (2009).

37. Stojkovic, B., Jovanovic, D. S., Tucic, B. \& Tucic, N. Homosexual behaviour and its longevity cost in females and males of the seed beetle Acanthoscelides obtectus. Physiol. Entomol. 35, 308-316 (2010).

38. Doebeli, M., Ispolatov, Y. \& Simon, B. Towards a mechanistic foundation of evolutionary theory. eLife 6, e23804 (2017).

39. Bailey, N. W. \& French, N. Same-sex sexual behaviour and mistaken identity in male field crickets, Teleogryllus oceanicus. Anim. Behav. 84, 1031-1038 (2012).

40. Macchiano, A., Razik, I. \& Sagot, M. Same-sex courtship behaviors in male-biased populations: evidence for the mistaken identity hypothesis. Acta Ethol. 21, 147-151 (2018).

41. Sales, K. et al. Experimental evolution with an insect model reveals that male homosexual behaviour occurs due to inaccurate mate choice. Anim. Behav. 139, 51-59 (2018).

42. Steiger, S. \& Muller, J. K. From class-specific to individual discrimination: acceptance threshold changes with risk in the partner recognition system of the burying beetle Nicophorus vespilloides. Anim. Behav. 80, 607-613 (2010).

43. Han, C. S. \& Brooks, R, C. Same-sex sexual behaviour as a byproduct of reproductive strategy under male-male scramble competition. Anim. Behav. 108, 193-197 (2015).

44. Engel, K. C., Manner, L., Ayasse, M. \& Steiger, S. Acceptance threshold theory can explain occurrence of homosexual behaviour. Biol. Letters 11, 20140603 (2015).

45. Reeve, H.K. The evolution of conspecific acceptance thresholds. Am. Nat. 133, 407-435 (1989).

46. Parker, G. A. The sexual cascade and the rise of pre-ejaculatory (Darwinian) sexual selection, sex roles, and sexual conflict. Cold Spring Harb. Perspect. Biol. 6, a017509 (2014).

47. Godin, J. J. \& Briggs, S. E. Female mate choice under predation risk in the guppy. Anim. Behav. 51, 117-130 (1996). 
48. Byers, J. A., Byers, A. A. \& Dunn, S. J. A dry summer diminishes mate search effort by pronghorn females: Evidence for a significant cost of mate search. Ethol. 112, 74-80 (2006).

49. Bluhm, B. A., Piepenburg, D. \& von Jeterzenka, K. Distribution, standing stock, growth, mortality, and production of Stronglyocentrotus pallidus (Echinodermata: Echinoidea) in the northern Barents Sea. Polar Biol. 20, 325-334 (1998).

50. Ebert, T. A., Russell, M. P., Gamba, G. \& Badnar, A. Growth, survival, and longevity estimates for the rock-boring sea urchin Echinometra lucunter lucenter (Echinodermata, Echinoidea) in Bermuda. Bull. Mar. Sci. 82, 381-403 (2008).

51. Miller, R. L. Evidence for the presence of sexual pheromones in free-spawning starfish. J. Exp. Mar. Biol. Ecol. 130, 205-221 (1989).

52. Mercier, A. \& Hamel, J. Endogenous and Exogenous Control of Gametogenesis and Spawning in Echinoderms Ch. 3: Spawning (Academic Press, 2009).

53. Slattery, M. \& Bosch, I. Mating behavior of a brooding Anarctic asteroid Neosmilaster georgianus. Invertebr. Reprod. Dev. 24, 97-102 (1993).

54. Bulmer, M. Structural instability of models of sexual selection. Theor. Pop. Biol. 35, 195208 (1989).

\section{Acknowledgements}

We thank Michael Moore and Max Lambert for comments on early versions of the manuscript and Aimee Deconinck for suggesting the name "targeted" sex.

\section{Author Contributions}

BAL conceived of the project and the optimization models. BAL and MRS designed the population genetic models. BAL led the writing on the manuscript with input from MRS.

\section{Data Availability}

All analyses can be reproduced by the files in the supplementary information.

\section{Competing Interests}

The authors declare no competing interests. 
369 Table 1. Conditions for no discrimination or complete discrimination to be the optimal

370 evolutionary strategy given an equal sex ratio for each of the models we consider.

\begin{tabular}{|l|c|c|c|}
\hline $\begin{array}{l}\text { Discrimination cost } \\
\text { to: }\end{array}$ & $\begin{array}{c}\text { Presented } \\
\text { in }\end{array}$ & $\begin{array}{c}\text { Indiscriminate mating } \\
\text { best strategy }\end{array}$ & $\begin{array}{c}\text { Complete sexual discrimination best } \\
\text { strategy }\end{array}$ \\
\hline Survival (additive) & Main text & $c>(1-d) d s$ & $c<\frac{-(1+2 d s)+\sqrt{1+4 d s(1+s)}}{2 s}$ \\
\hline $\begin{array}{l}\text { Survival } \\
\text { (multiplicative) }\end{array}$ & $\begin{array}{c}\text { Appendix } \\
\text { S2 }\end{array}$ & $c>d s$ & $c<\frac{-(1+2 d s)+\sqrt{1+4 d s(1+s)}}{2 s(1-d)}$ \\
\hline Fecundity & $\begin{array}{c}\text { Appendix } \\
\text { S3 }\end{array}$ & $c>s(1-p s)$ & $c<\frac{s(1-p s)}{1+2 s}$ \\
\hline $\begin{array}{l}\text { Survival (additive) with } \\
\text { semelparous life history }\end{array}$ & $\begin{array}{c}\text { Appendix } \\
\text { S4 }\end{array}$ & $c>(1-d) s$ & $s(1-d)$ \\
\hline $\begin{array}{l}\text { Survival (additive) with } \\
\text { extra cost to SSB }\end{array}$ & $\begin{array}{c}\text { Appendix } \\
\text { S1 }\end{array}$ & $\begin{array}{c}c>s[d((1-\delta)-d) \\
+(1-\delta / 4) \delta]\end{array}$ & $\begin{array}{c}c<-(1+2 d s+s \delta(1-s)) / 2 s+ \\
\sqrt{1+4 d s(1+s)+2 s \delta(1+s)} / 2 s\end{array}$ \\
\hline
\end{tabular}


373 Table 2. Summary of parameters, their meaning, and their role in the evolution of sexual

374 discrimination from the first model presented with discrimination as an additive cost to survival.

\begin{tabular}{|c|c|c|c|}
\hline Parameter & Meaning & Range & Effect \\
\hline$a$ & Attempt to mate discriminately & $0 \leq a \leq 1$ & N/A \\
\hline$c$ & Cost of mating discriminately & $0 \leq c \leq(1-d)$ & $\begin{array}{l}\text { Decreasing } c \text { favors sexual } \\
\text { discrimination }\end{array}$ \\
\hline$s$ & $\begin{array}{l}\text { Signal (by targeted sex) of their } \\
\text { sexual identity }\end{array}$ & $0 \leq s \leq 1$ & $\begin{array}{l}\text { Increasing } s \text { favors sexual } \\
\text { discrimination }\end{array}$ \\
\hline$\sigma$ & $\begin{array}{l}\text { Proportion of population of the } \\
\text { targeted sex }\end{array}$ & $0<\sigma<1$ & $\begin{array}{l}\text { Decreasing } \sigma \text { favors sexual } \\
\text { discrimination }\end{array}$ \\
\hline$p$ & $\begin{array}{l}\text { Cost of presenting sexual } \\
\text { identity (by targeted sex) }\end{array}$ & $0 \leq p<1$ & $\begin{array}{l}\text { Increasing } p \text { decreases the } \\
\text { selection gradient but has little } \\
\text { effect on the ESS }\end{array}$ \\
\hline$d$ & $\begin{array}{l}\text { Baseline mortality probability } \\
\text { between each reproductive bout }\end{array}$ & $0<d<1$ & $\begin{array}{l}\text { Intermediate values of } d \text { favor } \\
\text { sexual discrimination }\end{array}$ \\
\hline$f$ & $\begin{array}{l}\text { Probability of finding any } \\
\text { individual with which to } \\
\text { attempt mating }\end{array}$ & $0<f \leq 1$ & $\begin{array}{l}\text { Increasing } f \text { increases the } \\
\text { selection gradient but has little } \\
\text { effect on the ESS }\end{array}$ \\
\hline$r$ & $\begin{array}{l}\text { Probability mate of correct sex } \\
\text { rejects focal individual }\end{array}$ & $0 \leq r<1$ & $\begin{array}{l}\text { Increasing } r \text { decreases the } \\
\text { selection gradient but has little } \\
\text { effect on the ESS }\end{array}$ \\
\hline
\end{tabular}


377 Table 3. Summary of how key parameters qualitatively influence optimal sexual discrimination

378 in each model variant considered. The four distinct variants are shown as columns and are

379 presented in the location indicated by italics. The information in the table should be read as in the

380 following example. For the top left cell in the main body of the table: in the model variant where

381 there are additive survival costs to discrimination, high sexual discrimination evolves with a low

382 discrimination cost $c$.

\begin{tabular}{|c|c|c|c|c|}
\hline \multirow[b]{3}{*}{ Parameter } & \multicolumn{4}{|c|}{ In the model variant with... } \\
\hline & $\begin{array}{l}\text { Survival cost } \\
\text { (additive) } \\
\text { Main text }\end{array}$ & $\begin{array}{c}\text { Survival cost } \\
\text { (multiplicative) } \\
\text { Appendix } S 2\end{array}$ & $\begin{array}{c}\text { Fecundity cost } \\
\text { Appendix S3 }\end{array}$ & $\begin{array}{c}\text { Semelparous life } \\
\text { history } \\
\text { Appendix } S 4\end{array}$ \\
\hline & \multicolumn{4}{|c|}{ High sexual discrimination evolves with... } \\
\hline Discrimination cost $c$ & Low & Low & Low & Low \\
\hline Sexual signal $s$ & High & High & High & High \\
\hline Proportion targeted $\sigma$ & Low & Low & Low & Low \\
\hline Signaling cost $p$ & No effect & No effect & Low & No effect \\
\hline Mortality $d$ & Intermediate & High & No effect & Low \\
\hline
\end{tabular}



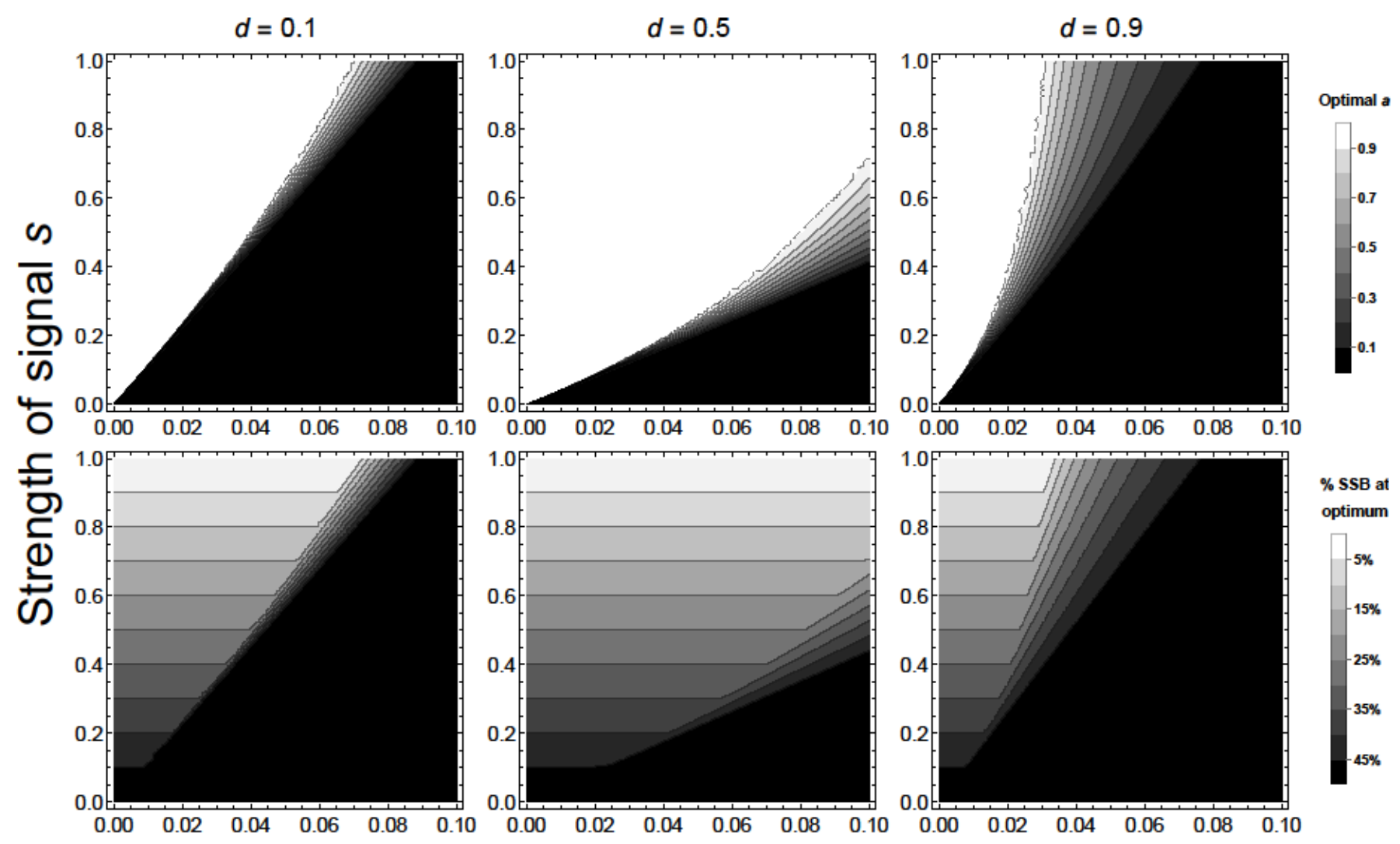

Fig. 1. Top row: Optimal discrimination strategies $a$ predicted by the optimization model with cost to discrimination $c$ on the x-axis, strength of sexual signal $s$ on the y-axis, and death rate $d$ increasing across columns from left to right. Higher levels of attempted discrimination correspond to lighter shading (white: $a=1$; black $a=0$ ). A wide range of parameter values predict that indiscriminate mating (black) is the best strategy. Indiscriminate mating is favored by increasing the cost of discrimination $c$ and decreasing the strength of the sexual signal $s$. Bottom row: proportion of matings expected to be SSB at the evolutionary optimum. Darker values indicate more same-sex matings (black: half of matings are with individuals of the same sex at the optimum; white: no matings are with individuals of the same sex if behaving optimally). Other parameters: proportion of the population of the targeted $\operatorname{sex} \sigma=0.5$, probability of finding any individual with which to attempt to mate $f=1$, probability of being rejected by a potential mate $r=0$, cost of sexual signal $p=0$. 